\title{
Microcirculatory perfusion disturbances and endothelial hyperpermeability following cardiopulmonary bypass persist in the early postoperative period
}

Nicole A.M. Dekker ${ }^{1,2}$, Anoek L.I. van Leeuwen ${ }^{1,2}$, Peter L. Hordijk ${ }^{2}$, Alexander B.A. Vonk ${ }^{3}$, Christa Boer ${ }^{1}$, Charissa E. van den Brom ${ }^{1,2}$ Departments of ${ }^{1}$ Anaesthesiology, ${ }^{2}$ Physiology and ${ }^{3}$ Cardiothoracic Surgery, Amsterdam Cardiovascular Sciences, VU University Medical Center Amsterdam, The Netherlands.

\section{Introduction}

Increased endothelial permeability following cardiopulmonary bypass (CPB) is an important contributor to microcirculatory perfusion disturbances and postoperative organ dysfunction. We therefore hypothesised that microcirculatory perfusion disturbances remain altered after surgery and are paralleled by prolonged impairment of endothelial barrier function.

\section{Methods}

Microcirculatory perfusion was assessed in 17 patients undergoing cardiac surgery with CPB using sublingual sidestream dark-field imaging from which red blood cell filling, percentage of perfused vessels, and perfused boundary region (glycocalyx permeability) was calculated. Plasma was obtained before connection to CPB (pre-CPB), during CPB, after weaning from CPB (post-CPB) and 1, 24 and 72 hours after weaning from CPB. Using electric cell-substrate impedance sensing (ECIS)of human pulmonary and renal microvascular endothelial cells (MVECs), the effect of plasma exposure for 3 hours on in vitro endothelial barrier was measured. Endothelial gap formation of pulmonary MVECs after plasma exposure was visualized using immunofluorescence (IF). Plasma syndecan-1 levels were studied with ELISA as marker for glycocalyx degradation.

\section{Results}

Patient characteristics

\section{$n=17$}

Age (years)

Males (\%)

Body mass index $\left(\mathrm{kg} / \mathrm{m}^{2}\right)$

Surgery time $(\mathrm{min})$

Cross-clamp time (min)

Packed red blood cell transfusion (\% patients)

Thrombocytes (5-donor concentrate; \% patients)

Cell saver retransfusion $(\mathrm{ml})$

Table 1. Values represent frequencies, means \pm standard deviation or median with interquartile range. ${ }^{\star} C P B$, cardiopulmonary bypass.

Prolonged impairment of microcirculatory parameters

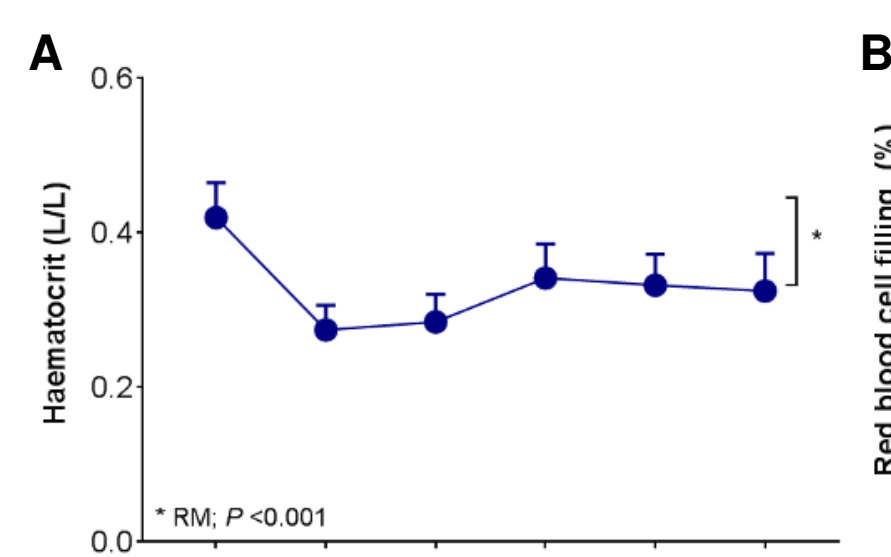

C

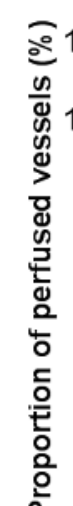

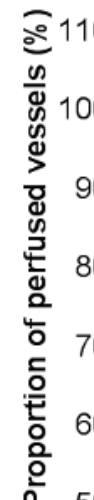
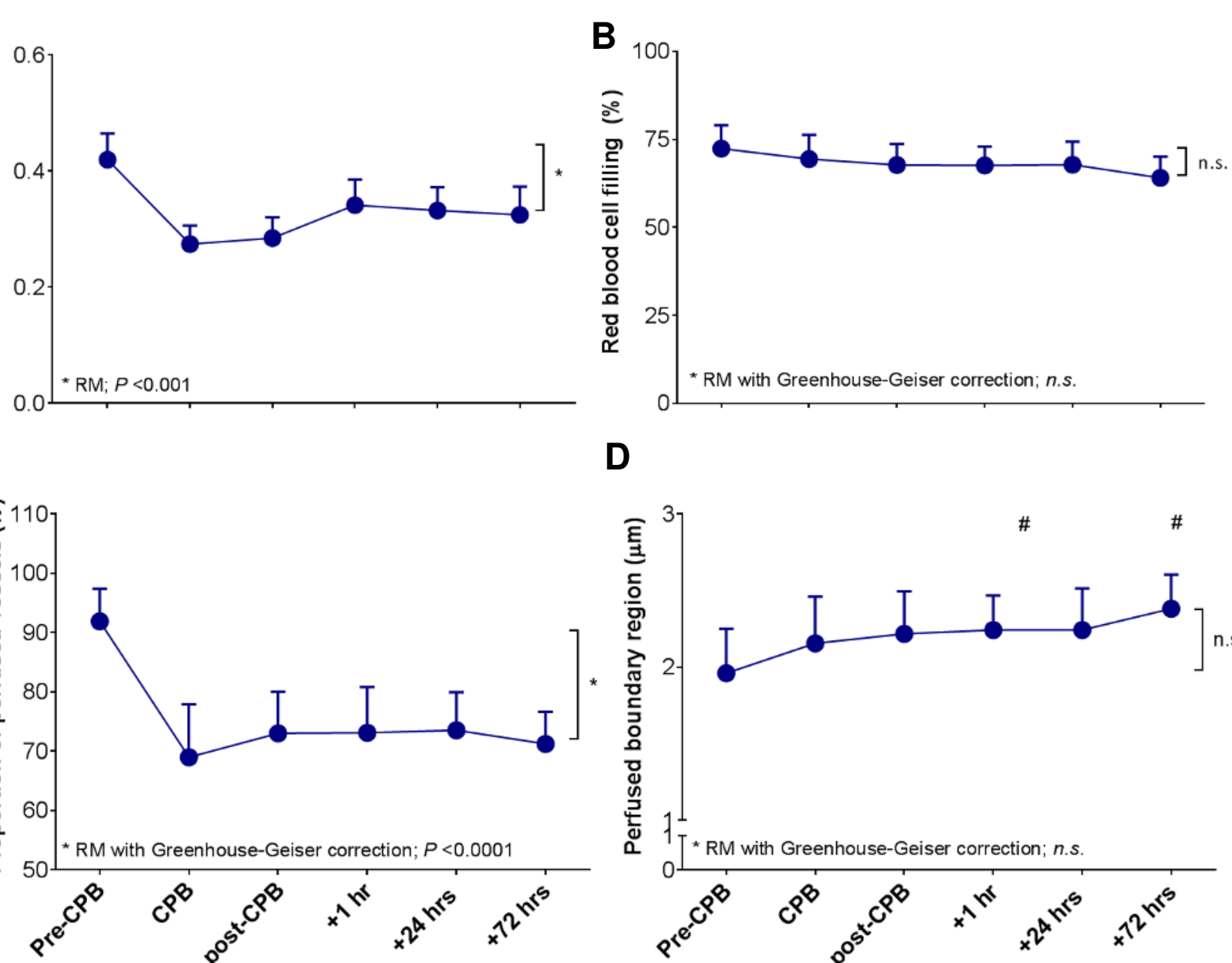

D

Fig. 1: Haematocrit levels $(\boldsymbol{A})$, capillary red blood cell filling (B), proportion of perfused vessels (C) and perfused boundary region (D) during the perioperative period. ${ }^{*} P<0.05$ RM ANOVA, \# $P<0.05$ vs. Pre-CPB.
Prolonged endothelial hyperpermeability and gap formation
A

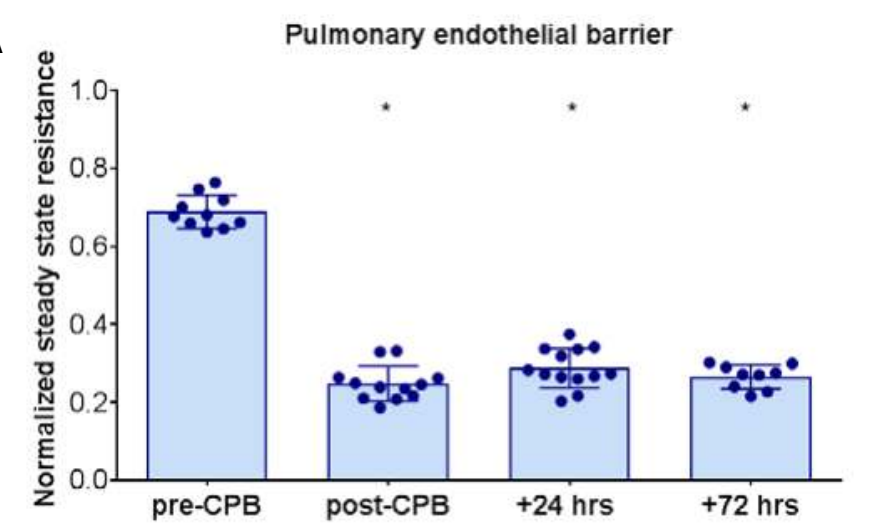

C

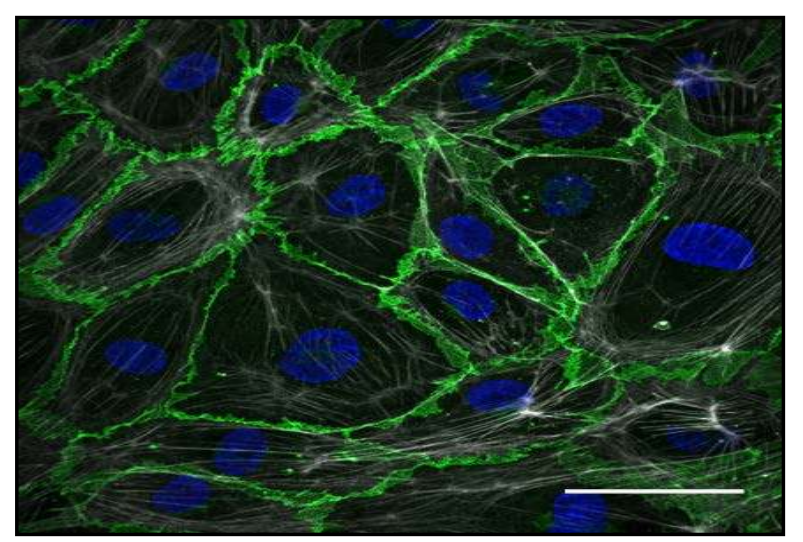

B.

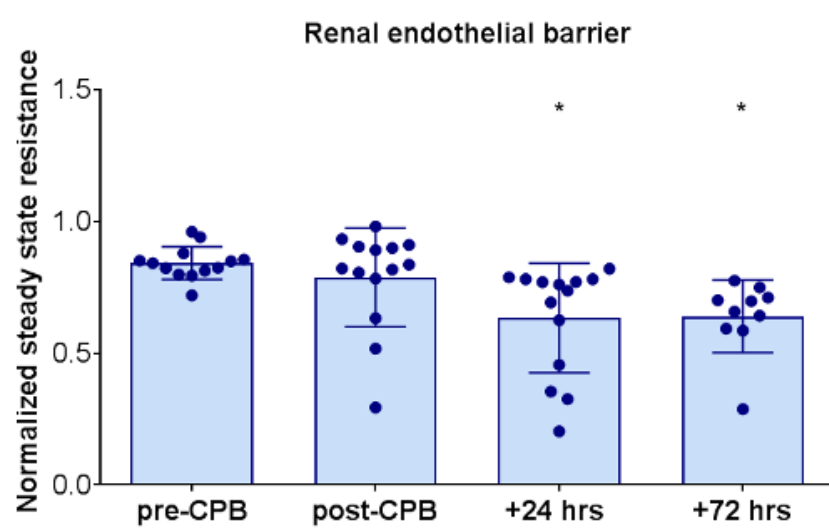

D

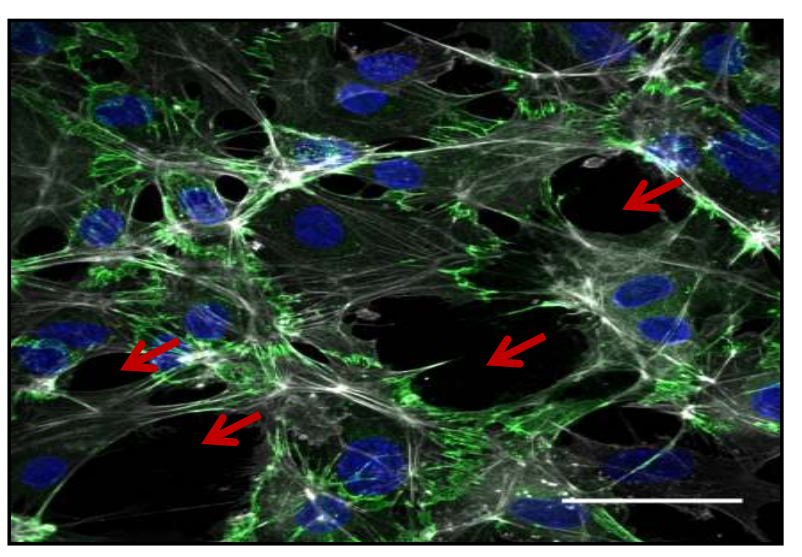

Fig. 2: Effect of plasma exposure obtained before and after CPB on pulmonary (A) and renal $(\boldsymbol{B})$ microvascular endothelial barrier measured with ECIS and IF staining of PMVECs exposed to pre-CPB (C) and post-CPB (D) plasma; VE-cadherin (green), DAPI (blue), F-actin (white); scale bar $50 \mu \mathrm{m}$. Endothelial gaps are indicated with red arrows * $P<0.05$ RM ANOVA.

Glycocalyx shedding is associated with microcirculatory perfusion disturbances and pulmonary endothelial hyperpermeability
A

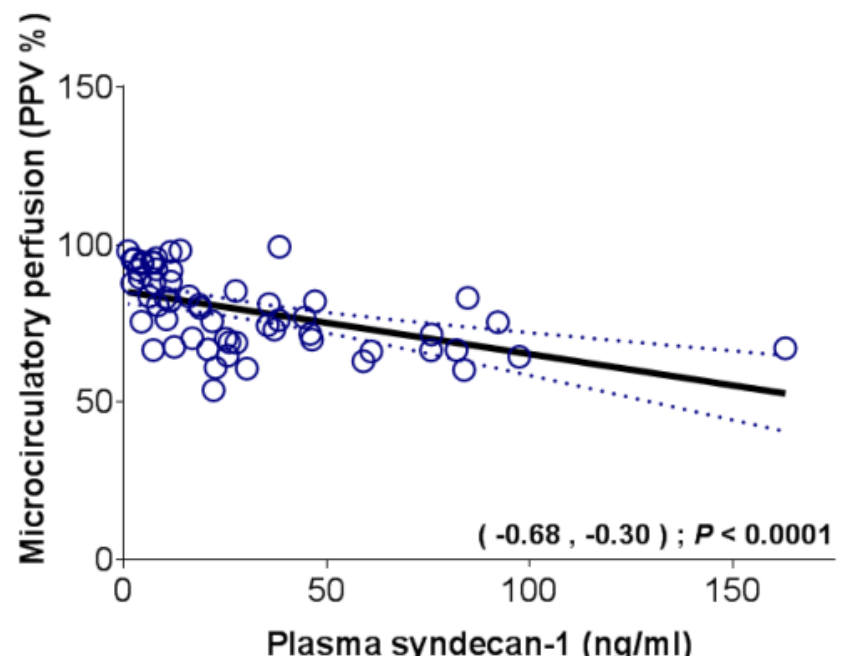

B

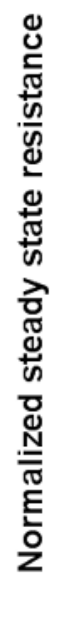

Fig. 3: Association between plasma glycocalyx shedding molecule syndecan-1 and proportion of perfused vessels (A) and pulmonary endothelial barrier (B). Pearson correlation. Values are presented with 95\% confidence intervals.

\section{Conclusion}

Microcirculatory perfusion disturbances in patients undergoing cardiac surgery with CPB persist in the first postoperative days and are paralleled by prolonged endothelial hyperpermeability and shedding of endothelial glycocalyx constituents. 